\title{
Valor pronóstico de la función renal a corto plazo en pacientes con infarto agudo de miocardio
}

\author{
Hedgar Berty Gutiérrez ${ }^{\mathrm{a}, *}$ y Frank Daniel Martos Benítez ${ }^{\mathrm{b}}$
}

\author{
a Unidad de Cuidados Intensivos, Hospital Universitario Clínico-Quirúrgico "Dr. Miguel Enríquez', La Habana, Cuba \\ b Hospital Universitario Clínico-Quirúrgico "Hermanos Almejeiras", La Habana, Cuba
}

Recibido el 7 de abril de 2017; aceptado el 11 de agosto de 2017

Disponible en Internet el 27 de noviembre de 2017

\section{PALABRAS CLAVE \\ Pronóstico; \\ Infarto agudo de \\ miocardio; \\ Filtrado glomerular; Insuficiencia renal}

\begin{abstract}
Resumen
Introducción: El infarto agudo de miocardio es una de las primeras causas de muerte en Cuba y el mundo. En la actualidad cerca de un 20 a 30\% de los pacientes con enfermedad coronaria, tienen insuficiencia renal.

Objetivo: Determinar la asociación entre insuficiencia renal en el momento del ingreso y la mortalidad a corto plazo en los pacientes con infarto agudo de miocardio.

Método: Se analizaron 284 pacientes consecutivos con diagnóstico de infarto agudo de miocardio ingresados en la Unidad de Cuidados Intensivos (UCI) del Hospital Universitario "Dr. Miguel Enríquez', entre el $1^{\circ}$. de enero 2015 y el $1^{\circ}$. de diciembre de 2016. Se determinó la creatinina, la tasa de filtrado glomerular estimado y las escalas de pronóstico cardiovascular en el momento del ingreso. Mediante regresión logística se evaluó la capacidad predictiva de muerte a corto plazo de la función renal.

Resultados: 26 pacientes fallecieron en la UCl (9,2\%). El valor de la creatinina sérica fue superior, en tanto que las tasas de filtrado glomerular fueron inferiores $(p<0,0001)$ en el grupo de pacientes que falleció respecto a los supervivientes. Todas las variables de función renal y las de pronóstico cardiovascular se asociaron con la mortalidad; al comparar ambas, se obtuvo una mejor discriminación con las primeras en relación a las segundas.

Conclusiones: La valoración de la función renal mediante la determinación de la creatinina y la estimación del filtrado glomerular, proporciona información útil y muy valiosa para la evaluación inicial de los pacientes con infarto agudo de miocardio.

(C) 2017 Sociedad Colombiana de Cardiología y Cirugía Cardiovascular. Publicado por Elsevier España, S.L.U. Este es un artículo Open Access bajo la licencia CC BY-NC-ND (http:// creativecommons.org/licenses/by-nc-nd/4.0/).
\end{abstract}

\footnotetext{
* Autor para correspondencia.

Correo electrónico: hedgar@infomed.sld.cu (H. Berty Gutiérrez).
} 


\section{KEYWORDS}

Prognostic;

Acute myocardial

infarction;

Glomerular filtrate;

Renal failure

\section{Short-term prognostic value of renal function in patients with acute myocardial infarction}

\begin{abstract}
Introduction: Acute myocardial infarction is one of the primary causes of death in Cuba, and the world. Currently, around $20 \%$ to $30 \%$ of patients with coronary disease have renal failure. Objective: To determine the relationship between renal failure at the time of admission and the short-term mortality in patients with acute myocardial infarction.

Material and methods: An analysis was made on a total of 284 consecutive patients with a diagnosis of acute myocardial infarction admitted into the Intensive Care Unit (ICU) of the "Dr. Miguel Enríquez"' University Hospital between 1 January 2015 and 1 December 2016. The creatinine and the calculated glomerular filtration rate were determined, as well as the scores on the cardiovascular prognostic scales, at the time of admission. The predictive value of the renal function for short-term death was evaluated using logistic regression.

Results: A total of $26(9.2 \%)$ patients died in the ICU. The serum creatinine was higher and the glomerular filtration rates were lower $(P<.001)$ in the patient group that died compared to the survivors. All the renal function variables and the cardiac prognostic scores were associated with mortality. A better discrimination was obtained with the renal function variables compared to the cardiovascular ones.

Conclusions: The assessment of renal function using the serum creatinine level and the calculated glomerular filtration rate provide very useful and valuable information for the initial evaluation of patients with acute myocardial infarction.

(c) 2017 Sociedad Colombiana de Cardiología y Cirugía Cardiovascular. Published by Elsevier España, S.L.U. This is an open access article under the CC BY-NC-ND license (http:// creativecommons.org/licenses/by-nc-nd/4.0/).
\end{abstract}

\section{Introducción}

La cardiopatía isquémica es una de las enfermedades de mayor prevalencia en las sociedades occidentales, catalogándose, junto con el resto de las enfermedades vasculares, como la primera causa de muerte en el mundo desarrollado. Su presentación como síndrome coronario agudo (SCA) reviste especial importancia, tanto por su morbimortalidad como por la cantidad elevada de recursos económicos, humanos y asistenciales que conlleva ${ }^{1}$.

En los Estados Unidos, casi 600.000 pacientes son hospitalizados cada año con un diagnóstico primario de SCA. La cifra supera el millón de pacientes si se incluye como diagnóstico secundario ${ }^{2,3}$. La incidencia aumenta considerablemente con la edad, tanto en hombres como en mujeres, y se observan también diferencias raciales (más frecuentes en hombres y mujeres negros, independientemente de la edad) $)^{2}$.

En Cuba, en el año 2015, la enfermedad cardiovascular (ECV) se mantuvo como la primera causa de muerte, con 24.497 defunciones, dentro de las cuales 7.183 representaron al infarto agudo de miocardio con una tasa de 64 por 100.000 habitantes ${ }^{4}$.

Existen varios factores que se han relacionado con un pronóstico desfavorable en los pacientes con ECV; uno de estos es la función renal, respecto a la cual en las últimas décadas se ha hecho más evidente la importancia de su correcta evaluación, sobre todo a raíz de estudios que han demostrado que esta relación directa entre función renal y acontecimientos cardiovasculares aparece ya en fases de disfunción renal moderada, e incluso, leve. La evaluación precisa de la función renal permite, por tanto, identificar de manera pre$\mathrm{coz}$ a los pacientes con riesgo elevado de acontecimientos cardiovasculares, con la finalidad de mejorar su pronóstico mediante una intervención oportuna de diagnóstico y tratamiento. Además, posibilita la monitorización estrecha de ciertas intervenciones como el ajuste de fármacos y la prevención de nefrotoxicidad por diversos agentes en pacientes de alto riesgo. Una mala estimación de la función renal puede llevar a que un paciente no reciba el tratamiento cardioprotector adecuado con base en su riesgo, hecho que conlleva aumento de la mortalidad ${ }^{5}$.

La creatinina ha sido clásicamente la sustancia endógena más utilizada para la evaluación de la función renal. No obstante, la concentración de creatinina se ve afectada por diversos factores (masa muscular, sexo, raza, tipo de alimentación), además de otros relacionados con la propia filtración de creatinina, como la secreción tubular, la producción y la excreción extrarrenal. Dadas las desventajas de la creatinina plasmática como marcador de deterioro renal, han sido múltiples los métodos propuestos para conocer la función renal, dentro de los cuales las fórmulas de estimación del filtrado glomerular constituyen el más usado en la práctica clínica habitual, dada su relativa sencillez ${ }^{5}$.

Existen diferentes fórmulas para estimar el filtrado glomerular en un determinado paciente. Todas ellas incorporan una serie de variables que intentan solventar las carencias de la creatinina plasmática como único marcador de función renal. Sin embargo, su precisión no es constante en todos los escenarios y sigue habiendo situaciones en las que no se tiene un método fiable para conocer el grado de deterioro renal real del paciente ${ }^{5}$. 
En Cuba no existen estudios que muestren la relación entre SCA y enfermedad renal, aunque en la literatura mundial hay trabajos sobre este tema en los que se asocian al infarto agudo de miocardio con el daño renal agudo y su repercusión en la mortalidad a largo plazo ${ }^{6,7}$. Este estudio, por ende, se llevó a cabo con el objetivo de determinar la influencia de la función renal sobre la mortalidad a corto plazo en pacientes con infarto agudo de miocardio.

\section{Materiales y métodos}

\section{Diseño}

Se realizó un estudio de cohorte, observacional, prospectivo en la Unidad de Cuidados Intensivos del Hospital Clínico Quirúrgico "Dr. Miguel Enríquez"', situado en la provincia La Habana, entre el $1^{\circ}$. de enero 2015 y el $1^{\circ}$. de diciembre de 2016. El centro cuenta con 430 camas en hospitalización y ocho camas en la UCl. El estudio fue aprobado con el Consejo Científico y el Comité de Ética del centro.

\section{Pacientes}

Se incluyeron 295 pacientes admitidos en la $\mathrm{UCl}$ con diagnóstico de infarto agudo de miocardio según la tercera definición universal de infarto de miocardio $(1219)^{8}$, ya sea infarto agudo de miocardio con elevación del segmento ST (IAMCEST) o infarto agudo de miocardio sin elevación del segmento ST (IAMSEST). Se excluyeron los sujetos con enfermedad renal crónica (ERC).

\section{Recolección de datos}

De la base de datos de la UCI se tomaron las siguientes variables al ingreso: edad, sexo, color de piel, historia de enfermedades crónicas, peso, clase Killip-Kimball (KK), riesgo de muerte intrahospitalaria según la escala GRACE ${ }^{9}$, realización de trombólisis y de intervención coronaria, creatinina, TFGe según la ecuación Chronic Kidney Disease Epidemiology Collaboration (CKD-EPI), Modification of Diet in Renal Disease (MDRD-4) y la Cockcroft-Gault (CG), así como la estadía en UCI. La variable de respuesta fue la mortalidad global en $\mathrm{UCl}$.

\section{Análisis estadístico}

Para todas las variables se utilizaron medidas de resumen, de manera que las variables cualitativas se muestran con números absolutos y porcentaje, mientras que las cuantitativas con mediana con 25-75\% rango intercuantílico (RIQ).

La comparación entre los grupos de supervivientes y fallecidos se realizó mediante la prueba de Chi cuadrado $\left(\chi^{2}\right)$ o el test exacto de Fisher. Para las variables cuantitativas se empleó la prueba $U$ de Mann-Whitney.

La influencia de las variables explicativas (pruebas de función renal y escalas de pronóstico cardiovascular) sobre la mortalidad en $\mathrm{UCl}$, se realizó mediante regresión logística, cuyos resultados se muestran en razón de disparidad (OR) con intervalo de confianza (IC) al 95\%. Las variables que no tenían una distribución normal, fueron transformadas previo al análisis, mediante logaritmo natural.

La bondad de ajuste del modelo fue valorada mediante la prueba de Hosmer-Lemeshow; un resultado significativo $(p<0,05)$ en esta prueba indica falta de ajuste del modelo. La capacidad de las pruebas de función renal para discriminar correctamente entre los casos que morirán y los que no, se evaluó mediante el área bajo la curva de las características operativas del receptor (ROC, por su sigla en inglés), también llamado estadístico $c$. Un valor del estadígrafo $c>0,70$ indica una capacidad de discriminación aceptable ${ }^{10}$.

Las pruebas estadísticas se consideraron significativas con un valor de $\mathrm{p}$ bilateral menor de 0,05. El análisis estadístico se realizó mediante el programa IBM ${ }^{\circledR}$ SPSS $^{\circledR}$, versión 23 (Chicago, IL, USA).

\section{Resultados}

\section{Características generales}

Durante el período de estudio se admitieron 410 pacientes con síndrome coronario agudo, de los cuales 295 presentaron infarto agudo de miocardio. Luego de excluir 9 pacientes con ERC, se analizaron 284 sujetos, 240 (84,5\%) con IAMCEST y 44 $(15,5 \%)$ con IAMSEST. Fallecieron en la UCI 26 sujetos $(9,2 \%)$.

En la tabla 1 se muestran las características generales de los pacientes. Se encontraron diferencias estadísticamente significativas en la distribución por edad $(p=0,001)$, sexo $(p=0,005)$, historia de diabetes mellitus $(p=0,017)$ e historia de enfermedad coronaria $(p=0,021)$ entre los pacientes fallecidos y los supervivientes. También se observó una asociación poderosa del IAMCEST $(p=0,009)$, de la clase Killip y Kimball $(p<0,0001)$ y de la categoría de riesgo de muerte intrahospitalaria según la escala GRACE $(p<0,0001)$ con la mortalidad. La intervención coronaria percutánea actuó como un factor de protección contra la muerte en $\mathrm{UCl}(\mathrm{p}=0,019)$.

\section{Función renal}

La mediana de la creatinina fue de 94,5 $\mu \mathrm{mol} / \mathrm{L}$ (RIQ 80,0 - 112,0 $\mu \mathrm{mol} / \mathrm{L})$. La TFGe (CKD-EPI), la TFGe (MDRD-4) y la TFGe (CG) tuvieron una mediana de $70,4 \mathrm{ml} / \mathrm{min} / 1,73$ $\mathrm{m}^{2}$ (RIQ 55,1 - 87,2 ml/ $\mathrm{min} / 1,73 \mathrm{~m}^{2}$ ), 72,5 ml/ $\mathrm{min} / 1,73$ $\mathrm{m}^{2}$ (RIQ $57,1-87,5 \mathrm{ml} / \mathrm{min} / 1,73 \mathrm{~m}^{2}$ ) y $67,6 \mathrm{ml} / \mathrm{min} / 1,73$ $\mathrm{m}^{2}$ (RIQ 52,7 - 88,5 ml/ $\mathrm{min} / 1,73 \mathrm{~m}^{2}$ ), respectivamente. En la tabla 2 se muestra la distribución de las características de la función renal entre los pacientes que fallecieron y los supervivientes. Se encontró que la distribución de la creatinina sérica fue significativamente superior en el grupo de pacientes que falleció respecto al grupo de supervivientes $(p<0,0001)$. Por su parte, la TFGe (CKD-EPI), la TFGe (MDRD-4) y la TFGe (CG) fueron significativamente inferiores entre los fallecidos en relación con los que no fallecieron $(\mathrm{p}<0,0001)$.

\section{Predicción de mortalidad}

En el análisis de regresión logística se encontró que todas las variables que evalúan la función renal, así como las 
Tabla 1 Características generales de los pacientes

\begin{tabular}{|c|c|c|c|}
\hline Variable & $\begin{array}{l}\text { Fallecidos } \\
n=26\end{array}$ & $\begin{array}{l}\text { Vivos } \\
n=258\end{array}$ & $\mathrm{p}$ \\
\hline Edad, mediana (RIQ) & $76,5(60,0-84,0)$ & $63,5(55,0-72,0)$ & 0,001 \\
\hline $\begin{array}{l}\text { Sexo, } n(\%) \\
\text { Masculino } \\
\text { Femenino }\end{array}$ & $\begin{array}{l}10(38,5) \\
16(61,5)\end{array}$ & $\begin{array}{r}171(66,3) \\
87(33,7)\end{array}$ & 0,005 \\
\hline $\begin{array}{l}\text { Color de piel, } n(\%) \\
\text { Negro } \\
\text { Blanco }\end{array}$ & $\begin{array}{l}11(42,3) \\
15(57,7)\end{array}$ & $\begin{array}{r}97(37,6) \\
161(62,4)\end{array}$ & 0,637 \\
\hline Peso, mediana $(R I Q)$ & $75,0(68,0-82,0)$ & $70,0(64,0-84,0)$ & 0,394 \\
\hline $\begin{array}{l}\text { Historia de enfermedades crónicas, } n \text { (\%) } \\
\text { Diabetes mellitus } \\
\text { Hipertensión arterial } \\
\text { Enfermedad coronaria } \\
\text { Tabaquismo }\end{array}$ & $\begin{array}{c}13(50,0) \\
25(96,2) \\
14(53,8) \\
1(4,0)\end{array}$ & $\begin{array}{r}71(27,5) \\
236(91,5) \\
81(31,4) \\
81(31,4)\end{array}$ & $\begin{array}{l}0,017 \\
0,706 \\
0,021 \\
0,089\end{array}$ \\
\hline $\begin{array}{l}\text { Tipo de IAM, } n(\%) \\
\text { IAMCEST } \\
\text { IAMSEST }\end{array}$ & $\begin{array}{r}17(65,4) \\
9(34,6)\end{array}$ & $\begin{array}{r}223(86,4) \\
35(13,6)\end{array}$ & 0,009 \\
\hline $\begin{array}{l}\text { Clase Killip-Kimball, } n(\%) \\
\text { I } \\
\text { II } \\
\text { III } \\
\text { IV }\end{array}$ & $\begin{aligned} 2 & (7,7) \\
11 & (42,3) \\
6 & (23,1) \\
7 & (26,9)\end{aligned}$ & $\begin{aligned} 209 & (81,0) \\
39 & (15,1) \\
6 & (2,3) \\
4 & (1,6)\end{aligned}$ & $<0,0001$ \\
\hline $\begin{array}{l}\text { Clase de riesgo de muerte intrahospitalaria, } n(\%) \\
\text { Bajo riesgo } \\
\text { Riesgo intermedio } \\
\text { Alto riesgo }\end{array}$ & $\begin{aligned} 1 & (3,8) \\
5 & (19,2) \\
20 & (76,9)\end{aligned}$ & $\begin{aligned} 179 & (69,4) \\
58 & (22,5) \\
21 & (8,1)\end{aligned}$ & $<0,0001$ \\
\hline $\begin{array}{l}\text { Trombólisis, } n(\%) \\
\text { Intervencionismo coronario, } n(\%) \\
\text { Estadía en UCI, mediana (RIQ) }\end{array}$ & $\begin{aligned} 7 & (26,9) \\
0 & (0,0) \\
2,0 & (1,5-5,0)\end{aligned}$ & $\begin{array}{l}78(30,2) \\
42(16,3) \\
3,0(2,0-4,0)\end{array}$ & $\begin{array}{l}0,725 \\
0,019 \\
0,611\end{array}$ \\
\hline
\end{tabular}

IAM: infarto agudo de miocardio; IAMCEST: IAM con elevación del segmento ST; IAMSEST: IAM sin elevación del segmento ST; RIQ: rango intercuartil; $\mathrm{UCl}$ : unidad de cuidados intensivos.

Tabla 2 Características de la función renal

\begin{tabular}{llll}
\hline Variable & Fallecidos & Vivos & p \\
& $\mathrm{n}=26$ & $\mathrm{n}=258$ & $<0,0001$ \\
\hline Creatinina, mediana (RIQ) & $161,5(132,0-190,0)$ & $92,0(79,0-108,0)$ & $<0,0001$ \\
TFGe (CKD-EPI), mediana (RIQ) & $34,9(23,2-39,5)$ & $72,6(58,8-90,0)$ & $<0,0001$ \\
TFGe (MDRD-4), mediana (RIQ) & $35,1(27,1-44,1)$ & $75,7(62,3-90,1)$ & $<0,0001$ \\
TFGe (CG), mediana (RIQ) & $34,0(25,3-44,1)$ & $69,6(56,2-92,0)$ & \\
\hline
\end{tabular}

CG: Cockcroft-Gault; CKD-EPI: Chronic Kidney Disease Epidemiology Collaboration; MDRD: Modification of Diet in Renal Disease; RIQ: rango intercuartil; TFGe: tasa de filtrado glomerular estimado.

variables de pronóstico cardiovascular, se asociaron significativamente con la mortalidad en UCI (tabla 3).

Al evaluar la capacidad predictiva, se observó una buena discriminación entre los pacientes que fallecieron y los supervivientes con todas las variables que evalúan la función renal (tabla 4 y fig. 1). Sin embargo, las mejores áreas bajo la curva ROC se observaron para la TFGe por la ecuación MDRD-4 y para la TFGe por la ecuación CKD-EPI, siendo de 0,981 y 0,980 , respectivamente.
Al comparar las variables que evalúan la función renal con las variables de pronóstico cardiovascular, se observó mejor discriminación con las primeras en relación a las segundas (tabla 4 y fig. 1).

\section{Discusión}

La presencia de insuficiencia renal es un conocido factor de mal pronóstico, tanto a corto como a largo plazo. En la 
Tabla 3 Resultados del análisis de regresión logística

\begin{tabular}{|c|c|c|c|c|}
\hline Variables & OR & $95 \%$ IC & $\mathrm{p}$ & $\begin{array}{l}\text { Hosmer-Lemeshow } \\
\left(\chi^{2} ; \mathrm{p} \text {-valor }\right)\end{array}$ \\
\hline Creatinina & 1,05 & $1,05-1,06$ & $<0,0001$ & 5,$03 ; 0,754$ \\
\hline TFGe (CKD-EPI) & 1,20 & $1,13-1,28$ & $<0,0001$ & 2,$12 ; 0,977$ \\
\hline TFGe (MDRD-4) & 1,20 & $1,13-1,28$ & $<0,0001$ & 2,$72 ; 0,951$ \\
\hline TFGe (CG) & 1,12 & $1,08-1,17$ & $<0,0001$ & 1,$93 ; 0,983$ \\
\hline Clase Killip-Kimball & & & & 0,$00 ; 1,000$ \\
\hline Clase I & Ref. & $(-)$ & $<0,0001$ & \\
\hline Clase II & 29,47 & $6,29-138,17$ & $<0,0001$ & \\
\hline Clase III & 104,50 & $17,37-628,61$ & $1<0,0001$ & \\
\hline Clase IV & 182,88 & $28,56-1171,1$ & 160,0001 & \\
\hline \multicolumn{5}{|c|}{ Categoría GRACE de riesgo de muerte intrahospitalaria } \\
\hline & & & & 0,$00 ; 1,000$ \\
\hline Bajo & Ref. & $(-)$ & $<0,0001$ & \\
\hline Intermedio & 15,43 & $1,77-134,79$ & 0,013 & \\
\hline Alto & 170,48 & $21,76-1335,7$ & $7 \ngtr 0,0001$ & \\
\hline
\end{tabular}

CG: Cockcroft-Gault; CKD-EPI: Chronic Kidney Disease Epidemiology Collaboration; IC: intervalo de confianza; MDRD: Modification of Diet in Renal Disease; OR: odds ratio; TFGe: tasa de filtrado glomerular estimado; $\chi^{2}$ : prueba $\mathrm{Chi}^{2}$

Tabla 4 Estadísticos de discriminación de la mortalidad para las pruebas de función renal y de pronóstico cardiovascular

\begin{tabular}{llll}
\hline Variable & $\mathrm{ABC}$ & $95 \% \mathrm{IC}$ & $\mathrm{p}$ \\
\hline Creatinina & 0,947 & $0,910-0,984$ & $<0,0001$ \\
TFGe (CKD-EPI) & 0,980 & $0,965-0,995$ & $<0,0001$ \\
TFGe (MDRD-4) & 0,981 & $0,966-0,995$ & $<0,0001$ \\
TFGe (CG) & 0,930 & $0,894-0,966$ & $<0,0001$ \\
Clase Killip-Kimball & 0,898 & $0,831-0,964$ & $<0,0001$ \\
Categoría GRACE de & 0,906 & $0,849-0,964$ & $<0,0001$ \\
riesgo de muerte & & & \\
intrahospitalaria & & & \\
\hline
\end{tabular}

$A B C$ : área bajo la curva ROC; CG: Cockcroft-Gault; CKD-EPI: Chronic Kidney Disease Epidemiology Collaboration; MDRD: Modification of Diet in Renal Disease; IC: intervalo de confianza; TFGe: tasa de filtrado glomerular estimado.

actualidad, de un $20-30 \%$ de los pacientes con enfermedad coronaria tienen insuficiencia renal ${ }^{11}$.

En este estudio se utilizaron como variables de función renal, la concentración sérica de creatinina y la estimación del filtrado glomerular mediante las ecuaciones CockcroftGault, MDRD-4 y CKD-EPI. Como resultado significativo se obtuvo un aumento de mortalidad en los pacientes con concentraciones elevadas de creatinina sérica o disminución de la TFGe en el momento del ingreso.

En el estudio Valsartan in Acute Myocardial Infarction Trial (VALIANT) se incluyeron pacientes con disfunción ventricular o insuficiencia cardiaca tras un infarto agudo de miocardio. Todos los eventos cardiovasculares importantes, como la mortalidad cardiovascular total, el infarto de miocardio, la insuficiencia cardiaca y el ataque cerebrovascular, estuvieron en estrecha relación con el grado de disfunción renal. La incidencia de dichos eventos se duplicó o triplicó en pacientes con filtrado glomeru- lar $<45 \mathrm{~mL} / \mathrm{min} / 1,73 \mathrm{~m}^{2}$ respecto a los que tenían un filtrado glomerular $>75 \mathrm{~mL} / \mathrm{min} / 1,73 \mathrm{~m}^{2}{ }^{12}$. En el estudio Survival and Ventricular Enlargement (SAVE), con un diseño similar, se obtuvieron los mismos resultados ${ }^{13}$.

En el análisis de regresión logística se encontró que todas las variables que evalúan la función renal, se asociaron de manera significativa con la mortalidad en UCI. Similares resultados se reflejan en el registro ACTION (Acute Coronary Treatment and Intervention Outcome Network), el cual evaluó a 57.700 casos con SCA y demostró que aproximadamente 1 de cada 6 pacientes con infarto agudo de miocardio presentaban lesión renal aguda, la cual se asoció con aumento de la mortalidad. Los pacientes con SCA tipo ST sin disfunción renal, tuvieron una mortalidad del $2 \%$ frente al $32 \%$ observado en los pacientes con falla renal grave ${ }^{11}$. De manera general, se logró una buena capacidad predictiva con todas las variables de función renal, siendo más exactas las TFGe obtenidas por la ecuación MDRD-4 y CKD-EPI. Estas son más precisas para evaluar las tasas de filtrado glomerular que la de Cockcroft-Gault, en la cual las estimaciones del peso o del peso ideal pueden complicar el cálculo y la presentación de los resultados ${ }^{14}$.

Al comparar las variables de pronóstico cardiovascular con las variables de función renal, se observó en estas últimas una mejor estratificación de riesgo. Con base en lo anterior, es importante señalar que en la estratificación pronóstica precoz tras un síndrome coronario, solamente se evalúa la función renal como factor de riesgo en el modelo del estudio $\mathrm{GRACE}^{9}$ y en el score de riesgo del estudio CADILLAC $^{15}$, mientras que en otras como en la escala TIMI $^{16}$ no se hace referencia alguna.

Las limitaciones de este trabajo son las propias de los estudios observacionales que no permiten una aleatorización estratificada, junto al hecho de que la determinación de la creatinina y por tanto de la TFGe en el momento del ingreso pudo verse modificada por las condiciones hemodinámicas iniciales del paciente grave y no refle- 


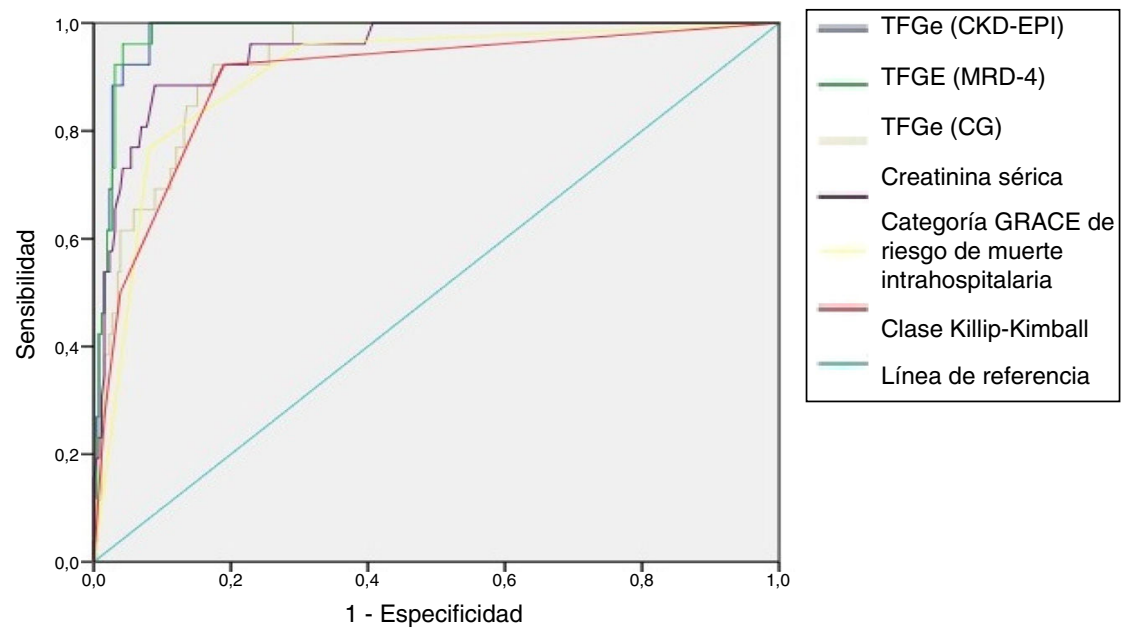

Figura 1 Área bajo la curva de las características operativas del receptor para las variables de función renal y pronóstico cardiovascular.

CG: Cockcroft-Gault; CKD-EPI: Chronic Kidney Disease Epidemiology Collaboration; MDRD: Modification of Diet in Renal Disease; TFGe: tasa de filtrado glomerular estimado.

jan fielmente la función renal basal, además el tamaño de la muestra seleccionada fue relativamente pequeño, lo cual explica la presencia de algunos intervalos con gran amplitud.

\section{Conclusiones}

La valoración de la función renal mediante la determinación de la creatinina y la estimación del filtrado glomerular, proporcionan información útil y muy valiosa para la evaluación inicial de los pacientes con infarto agudo de miocardio con o sin elevación del ST. Cualquier incremento en las concentraciones de creatinina o disminución del filtrado glomerular, refleja un aumento de la probabilidad de muerte a corto plazo en los pacientes con diagnóstico de infarto agudo de miocardio. Se trata, por tanto, de un marcador de obtención inmediata, fácil y disponible en todos los centros de salud.

\section{Responsabilidades éticas}

Protección de personas y animales. Los autores declaran que para esta investigación no se han realizado experimentos en seres humanos ni en animales.

Confidencialidad de los datos. Los autores declaran que en este artículo no aparecen datos de pacientes.

Derecho a la privacidad y consentimiento informado. Los autores declaran que en este artículo no aparecen datos de pacientes.

\section{Conflictos de interés}

Ninguno.

\section{Bibliografía}

1. Parra Fuertes PJ, de la Fuente SI. Síndrome coronario agudo sin elevación persistente del segmento ST. En: Montejo CJ, de Lorenzo GA, Marco P, Ortiz C, editores. Manual de medicina intensiva. $5^{\text {ta }}$ edición. Barcelona: Elsevier; 2017. p. 105-9.

2. Scirica BM, Morrow DA. Infarto de miocardio con elevación del ST: anatomía patológica, fisiopatología y manifestaciones clínicas. En: Braunwald E, editor. Braunwald Tratado de Cardiología. 10a. edición Barcelona: Elsevier; 2016. p. 92-1068.

3. Go AS. Heart disease and stroke statistics-2013 update: a report from the American Heart Association. Circulation. 2013;127. E841-E.

4. Estadísticas de Salud [Internet]. (Acceso 05 Ene 2017). Disponible en: http://www.sld.cu/sitios/dne/.

5. Flores Blanco PJ. Papel pronóstico de nuevos métodos de valoración de la función renal en insuficiencia cardíaca aguda y síndrome coronario agudo sin elevación del segmento ST. 2016.

6. Amin AP, Spertus JA, Reid KJ, Lan X, Buchanan DM, Decker C, et al. The prognostic importance of worsening renal function during an acute myocardial infarction on long-term mortality. American heart journal. 2010;160:71-1065.

7. Parikh CR, Coca SG, Wang Y, Masoudi FA, Krumholz HM. Longterm prognosis of acute kidney injury after acute myocardial infarction. Arch Int Med. 2008;168:987-95.

8. Thygesen K, Alpert JS, Jaffe AS, Simoons ML, Chaitman BR, White HD, et al. Third universal definition of myocardial infarction. Eur Heart J. 2012;33:67-2551.

9. Eagle KA, Lim MJ, Dabbous OH, Pieper KS, Goldberg RJ, Van de Werf $F$, et al. A validated prediction model for all forms of acute coronary syndrome: estimating the risk of 6 month postdischarge death in an international registry. JAMA. 2004;291:33-2727.

10. Betancourt-Plazaa I, Martos-Benítezb FD. TIMI score as a predictor of death in patients with acute myocardial infarction without percutaneous coronary intervention. CORSALUD. 2016;8:111-6.

11. Comignani P, Gambarte G. Insuficiencia renal en situaciones vinculadas con la Unidad Coronaria. PROSAC. Módulo 7,Fascículo. 2011;2:37-46. 
12. Anavekar NS, McMurray JJ, Velazquez EJ, Solomon SD, Kober $\mathrm{L}$, Rouleau J-L, et al. Relation between renal dysfunction and cardiovascular outcomes after myocardial infarction. New Eng J Med. 2004;351:1285-95.

13. Jose P, Skali H, Anavekar N, Tomson C, Krumholz HM, Rouleau JL, et al. Increase in creatinine and cardiovascular risk in patients with systolic dysfunction after myocardial infarction. J Am Soc Nephrol. 2006;17:2886-91.

14. Landry DW, Bazari H. Aproximación al paciente con enfermedad renal. En: Goldman-Cecil, editor. Tratado de medicina
interna+Expert Consult. 25a Edición Barcelona: Elsevier; 2017. p. 728-36.

15. Halkin A, Singh M, Nikolsky E, Grines CL, Tcheng JE, Garcia E, et al. Prediction of mortality after primary percutaneous coronary intervention for acute myocardial infarction: the CADILLAC risk score. J Am Coll Cardiol. 2005;45:1397-405.

16. Sánchez A, Pou M, Leiro R, Gómez DL, Ruiz MM, Saurina A, et al. El grado de insuficiencia renal como predictor de mortalidad tras un síndrome coronario agudo. Nefrología. 2009;29: 53-60. 